\title{
52. On some Fundamental Theorems in the Theory of Operators in Hilbert Space.
}

\author{
By Kunihiko KodaIRA. \\ Mathematical Institute, Tokyo Imperial University. \\ (Comm. by T. TaKagI, M.I.A., July 12, 1939.)
}

The purpose of this note is to point out that some fundamental theorems in the theory of operators in Hilbert space, viz.

I. the possibility of the canonical decomposition of closed linear operators with an everywhere dense domain ;')

II. the possibility of the integral representation of normal and especially self adjoint operators ${ }^{2}$

are easily deducible from a certain lemma contained in the proofs of the lemmas 9.1.2, 9.1.3, 9.1.4 in R.)

We state this lemma in $\S 1$, and then prove these fundamental theorems in the following two paragraphs. The sole knowledge presupposed to our demonstrations is that of operational calculus for bounded operators exposed for example in $\mathrm{E}^{2}$, Anhang III. Our proofs need not be altered, if we have to consider the generalized complex Euclidean space instead of the Hilbert space, as we make no use of the separability or of the infinite dimensionality of the space. $\$ \S 1,2$ hold good also for the generalized real Euclidean space, so that our proof for I has somewhat larger validity than von Neumann's given in A. ${ }^{3)}$

1. Lemma. Let $\mathfrak{A}$ be a linear, everywhere dense set in Hilbert space (or in generalized Euclidean space) $\mathfrak{S}:[\mathfrak{A}]=\mathfrak{S}$, and $Q(f, g)$ be a complex-valued (or real-valued, if one has to consider the real Euclidean space) function of $f, g \in \mathfrak{A}$, having the properties of inner product in I. We suppose that $\mathscr{A}$ is Q-complete, i. e. complete with respect to the metric determined by $Q(f, f)$. If $Q(f, f) \geqq(f, f)$ for all $f \in \mathfrak{A}$, then there exists a unique operator $B$ in $\mathfrak{S}$ mapping $\mathfrak{S}$ in $\mathfrak{A}$ so that

$$
Q(B f, g)=(f, g) \text { for } f \in \mathfrak{S}, \quad g \in \mathfrak{A} \text {. }
$$

$B$ has the following properties:

1) $B$ is a bounded Hermitian operator and $0<(B f, f) \leqq(f, f)$ if $f \neq 0$; so that we can form operators as $\sqrt{B}, \sqrt{1-B}$ in the sense of $F$. Riesz.

2) $\mathfrak{A}=$ Range $\sqrt{B}$

3) $Q(\sqrt{B} f, \sqrt{B} g)=(f, g)$ for all $f, g \in \mathfrak{S}$.

1) J. v. Neumann: Über adjungierte Funktionaloperatoren, Ann. of Math. 33 (quoted as A). See also F. J. Murray and J. v. Neumann: On rings of operators, Ann. of Math. 37 (quoted as R.) especially p. 141-142. As to the notation and terminology we follow the usage in $R$.

2) J. v. Neumann: Allgemeine Eigenwerttheorie Hermitescher Funktionaloperatoren, Math. Ann. 102 (quoted as E.) and Zur Algebra der Funktionaloperatoren, ibid

3) We wish to remark, by the way, that we could verify that all results of $R$. hold with slight modifications for complex or real generalized Euclidean space. We reserve it for later publications. 
We have not to dwell upon the proof of this Lemma, as it is fully contained in the proofs of above quoted lemmas in $\mathrm{R}^{4)}$ The following consequences are of importance for us.

2) and 3) imply that $\sqrt{B}$ maps $\mathfrak{S}$ isometrically on $\mathfrak{A}$, (when the latter is metrized with $Q$ ). So we can form the inverse $\sqrt{B^{-1}}$ of $\sqrt{\bar{B} \text {. }}$ Let us now consider the operator

$$
H=\sqrt{1-B} \sqrt{B^{-1}} .
$$

$H$ has evidently the domain $\mathfrak{A}$. We will show that it is self adjoint and semi-definite.

Remark, to the purpose, that each $f \in \mathfrak{A}$ can be put in the form $f=\sqrt{B f_{1}}$. If $g^{*}=H g$, we have therefore $(H f, g)=\left(\sqrt{1-B} f_{1}, g\right)=$ $\left(f_{1}, \sqrt{1-B} \sqrt{ } \bar{B} \sqrt{B^{-1}} g\right)=\left(f_{1}, \sqrt{B H} g\right)=\left(\sqrt{B} f_{1}, g^{*}\right)=\left(f, g^{*}\right)$ for all $f \in \mathfrak{A}$. Conversely, suppose $(H f, g)=\left(f, g^{*}\right)$ for all $f \in \mathfrak{A}$, and some $g, g^{*}$. Put $f=\sqrt{B} f_{1}$, then we have $\left(\sqrt{1-B} f_{1}, g\right)=\left(\sqrt{B} f_{1}, g^{*}\right)$, or $\left(f_{1}, \sqrt{1-B g}\right)$ $=\left(f_{1}, \sqrt{B} g^{*}\right)$ for all $f_{1} \in \mathfrak{G}$. Therefore $\sqrt{1-\bar{B} g}=\sqrt{B} g^{*},(1-B) g=$ $\sqrt{1-B} \sqrt{B g^{*}}$ or $g=\sqrt{B}\left(\sqrt{B g}+\sqrt{1-B g}{ }^{*}\right)$. So we have $g \in \mathfrak{A}$ by 2$)$, and $H g=\sqrt{1-B}\left(\sqrt{B g}+\sqrt{1-B} g^{*}\right)=\sqrt{B} \sqrt{1-B g}+(1-B) g^{*}=g^{*} . \quad H$ is thus self adjoint. $H$ is positive semi-definite, as $(H f, f)=\left(\sqrt{1-B f_{1}}\right.$ $\left.\sqrt{B} f_{1}\right)=\left(\sqrt{B} \sqrt{1-B} f_{1}, f_{1}\right) \geqq 0$ by 1$)$.

2. Canonical decomposition. Let $A$ be a linear closed operator with an everywhere dense domain $\mathfrak{A}$. Let us put, after K. Friedrichs ${ }^{5)}$

$$
Q(f, g)=(A f, A g)+(f, g) \text {. }
$$

One verifies immediately that this $Q(f, g)$ satisfies the conditions of the lemma. (In particular, $\mathfrak{A}$ is $Q$-complete, because $A$ is closed.) We may therefore write in virtue of the lemma

$$
\left(A \sqrt{ } \bar{B} f_{1}, A \sqrt{B} g_{1}\right)+\left(\sqrt{B} f_{1}, \sqrt{B} g_{1}\right)=\left(f_{1}, g_{1}\right) \text { for all } f_{1}, g_{1} \in \mathfrak{G} \text {. }
$$

Now we have $\left(f_{1}, g_{1}\right)-\left(\sqrt{B} f_{1}, \sqrt{B g_{1}}\right)=\left(f_{1}, g_{1}\right)-\left(B f_{1}, g_{1}\right)=\left((1-B) f_{1}, g_{1}\right)$ $=\left(\sqrt{1-B} f_{1}, \sqrt{1-B} g_{1}\right)$, so that we obtain $\left(A \sqrt{B} f_{1}, A \sqrt{B} g_{1}\right)=\left(\sqrt{1-B} f_{1}\right.$, $\sqrt{1-B g_{1}}$ ) and specially $\|A f\|=\|H f\|$, if we use the notation (2) and put $\sqrt{ } \bar{B} f_{1}=f$. In putting

$$
A=W H \text {, }
$$

we define therefore a partially isometric operator $W$ with the initial set

[Range $H]=\mathfrak{E}-(f ; H f=0)=\mathfrak{E}-(f ; A f=0)=\left[\right.$ Range $\left.A^{*}\right]$

and the final set

$$
\text { [Range } A]=\mathfrak{L}-\left(f ; A^{*} f=0\right) \text {. }
$$

As $H$ is self adjoint, one derives easily from (4)

$$
A^{*}=H W^{*} \text {. }
$$

4) The proofs in $R$. are valid also for complex or real generalized Euclidean space, for the Riesz's theorem holds, as is well-known, also for the non-separable case. Cf. F. Rellich: Spektraltheorie in nichtseparablen Räumen, Math. Ann. 110.

5) K. Friedirchs : Spektraltheorie halbbeschränkter Operatoren, Math. Ann. 109, 
3. Integral representation. We will adopt the following definition of the normal operator ${ }^{6}$ : a linear operator $A$ with an everywhere dense domain $\mathfrak{A}$ is normal, if $A^{*}$ has the same domain $\mathfrak{A}$ as $A$, and $(A f, A g)=\left(A^{*} f, A^{*} g\right)$ for all $f, g \in \mathfrak{A} .^{?)}$

Let $B, H, W$ be the operators defined by (3), (1), (2), (4), in the the preceding paragraphs for a normal operator $A$. We will show that $B$ commutes with $W$.

As $A f=0$ and $A^{*} f=0$ are equivalent in this case, we have [Range $A]=\left[\right.$ Range $\left.A^{*}\right]=$ [Range $\left.H\right] . W^{*} W$ and $W W^{*}$ being the projection of this closed linear set, they are equal and we have $W^{*} W H=H$. As $H$ is self adjoint, [Range $H]=\mathfrak{E}-(f, H f=0)$; we have also $H=H \cdot 1=H\left(1-W^{*} W\right)+H W^{*} W=H W^{*} W ;$ therefore $Q\left(W^{*} f, g\right)=$ $\left(A W^{*} f, A g\right)+\left(W^{*} f, g\right)=\left(H W^{*} f, H g\right)+\left(W^{*} f, g\right)=\left(H W^{*} f, H W^{*} W g\right)+$ $\left(W^{*} f, g\right)=\left(A^{*} f, A^{*} W g\right)+\left(W^{*} f, g\right)=(A f, A W g)+(f, W g)=Q(f, W g)$, and further $Q\left(W^{*} B f, g\right)=Q(B f, W g)=(f, W g)=\left(W^{*} f, g\right)=Q\left(B W^{*} f, g\right)$ for all $g \in \mathfrak{A}$; thus $W^{*} B=B W^{*}$ and consequently $W B=B W$.

Now $A$ can be put in the form

$$
A=U H,
$$

where $U$ is a unitary operator. It suffices to put: $U=W+\left(1-W^{*} W\right)$ as $W^{*} W=W W^{*}$. It is then clear that $B$ commutes also with $U$.

Let

$$
B=\int_{0}^{1} \mu d E_{B}(\mu), \quad U=\int_{0}^{2 \pi} e^{i \theta} d F(\theta)
$$

be the integral representations of $B$ and $U$ respectively. It is easily seen that the spectrum of $H$ is obtained from that of $B$ by the transformation $\rho=\sqrt{1-\mu} \sqrt{\mu^{-1}}$ or $\mu=\frac{1}{1+\rho^{2}}$; i. e.

$$
H=\int_{0}^{\infty} \rho d E_{H}(\rho)
$$

in an appropriate sense, where $E_{H}(\rho)=1-E_{B}\left(\frac{1}{1+\rho^{2}}\right)$.

$E_{H}(\rho)$ and $F(\theta)$ commute with each other, as $B$ and $U$ do so. Put

$$
E_{A}(\rho, \theta)=E_{H}(\rho) F(\theta), \quad 0 \leqq \rho<\infty, 0 \leqq \theta<2 \pi ;
$$

then we have clearly :

and

a) $E_{A}(\rho, \theta) \cdot E_{A}\left(\rho^{\prime}, \theta^{\prime}\right)=E_{A}\left(\rho^{\prime}, \theta^{\prime}\right) \cdot E_{A}(\rho, \theta)=E_{A}\left(\min \left(\rho, \rho^{\prime}\right), \min \left(\theta, \theta^{\prime}\right)\right)$

B) $\lim _{\substack{\theta \rightarrow 2 \pi \\ \rho \rightarrow \infty}} E_{A}(\rho, \theta)=1$

$$
A=U H=\int_{0}^{\infty} \int_{0}^{2 \pi} \rho e^{i \theta} d E_{A}(\rho, \theta) .
$$

The uniqueness of this representation can be also shown in the usual way.

6) See A., Satz 9.

7) $A$ is then clearly closed. 
In particular, when $A$ is self adjoint, i. e. $A=A^{*}, W H=H W^{*}$, we have $W H \sqrt{B}=H W^{*} \sqrt{B}=H \sqrt{B} W^{*}$, and so $W \sqrt{1-B}=\sqrt{1-B} W^{*}$ $W^{*} \sqrt{1-B}$. Then we get $W=W^{*}$. Indeed $W W^{*}=W^{*} W$ being, as already remarked, the projection of [Range $H]=[$ Range $\sqrt{1-B}]$, it follows $W=W W^{*} W=W^{*} W^{*} W=W^{*} W W^{*}=W^{*} . \quad W^{2}=W W^{*}$ is therefore a projection. Put $E^{+}=\frac{1}{2}\left(W^{2}+W\right), E^{-}=\frac{1}{2}\left(W^{2}-W\right)$. The spectrum of $A$ is then clearly constituted by

$$
\begin{cases}E(-\lambda)=E^{-}\left(1-E_{H}(\lambda)\right) & \text { for } \lambda>0, \\ E(\lambda)=1-E^{+}+E^{+} E_{H}(\lambda) & \text { for } \lambda \geqq 0 ;\end{cases}
$$

i. e.

$$
A=\int_{-\infty}^{\infty} \lambda d E(\lambda)
$$

$E(\lambda)$ being defined by (5).

If, moreover, $A$ is positive semi-definite, we must have $E^{-}=0$, $A=H$. As $H$ is uniquely determined by $A$ by (3), (1), (2), we see that the canonical decomposition of a linear closed operator is possible in a unique manner. 